\title{
Interdependence of marital status and clinical characteristics of morbidity with health-related quality of life among low back pain patients
}

\author{
Adebisi I. Hammed, Elvis I. Agbonlahor \\ Department of Human Kinetics and Sports Science, University of Benin, Benin City
}

\section{Summary}

Study aim: This study investigated the relationship of clinical characteristics of morbidity (CCM) and marital status with health-related quality of life (HRQoL) among patients with low back pain (LBP).

Material and methods: A total of 100 subjects with LBP of mechanical origin participated in this study. HRQoL of the participants was measured with the short form-36 (SF-36) questionnaire and CCM were categorized based on duration of onset of LBP in weeks into acute ( $<6$ weeks), sub-acute (6-12 weeks) and chronic ( $>12$ weeks). The relationship of CCM and marital status with HRQoL was analysed using Pearson's product moment coefficient of correlation.

Results: The outcome of this study showed that CCM correlated poorly and inversely with all domains of HRQoL except mental health, though that was insignificant $(\mathrm{p}>0.05)$. Also, a proportionate but insignificant relationship was found between marital status and domains of HRQoL except physical functioning and role limitations due to physical health problems.

Conclusion: It was therefore concluded that CCM and marital status cannot determine or predict HRQoL among LBP patients, and it might not be necessary to take them into consideration during rehabilitation of these individuals.

Keywords: Duration of LBP onset - Marital status - Quality of life - Low back pain

\section{Introduction}

Low back pain (LBP) is an important clinical, social, economic and public health problem affecting the population indiscriminately, i.e. it affects almost everyone in life, men and women equally across various strata of the society from laymen in the street to health care providers in health institutions. It is a disorder with many possible aetiologies, and its impact is pervasive. However, the vast literature available on LBP is not only heterogeneous but also contradictory. The Global Burden of Disease (GBD) estimated that LBP is among the top ten DALYs (disability-adjusted life years) causing diseases and injuries. Moreover, it is a major cause of disability that affects work performance and well-being. It can however be acute, subacute or chronic [7]. Across the populace, the elderly, sedentary workers including chief executives, professional drivers and weight lifters are all apt to develop LBP at one time or another [20]. LBP is a symptom that cannot be validated by an external standard, the symptom being what a person reports [14].
Consequently, LBP is rarely fatal but profoundly affects functioning, so the health-related quality of life (HRQoL) of the patients with LBP is significantly lower than healthy persons in the physical and psychological domains. Although there were significant correlations of HRQoL with pain intensity and lumbosacral radiculopathy, it is more important to improve the disability status rather than simple pain relief for the promotion of HRQoL [9]. The results further revealed that patients with LBP suffer not only from physical discomfort but also functional limitation that might cause disability and interfere with their quality of life (QoL). Acute LBP is usually defined as the duration of an episode of LBP persisting for less than 6 weeks, sub-acute LBP persists between 6 and 12 weeks, and chronic LBP persists for 12 weeks or more [26]. Furthermore, chronic LBP is the most common complaint of the working-age population and hampers the QoL, causing physical limitation and psychological distress, which may sometimes lead to disability. In addition to human suffering, it causes a substantial economic burden due to the wide use of medical services and absence from work [19]. Although in most patients acute LBP resolves with 
conservative treatment or without any treatment, the back pain appears to recur and becomes chronic more often than expected $[9,11]$. It has also been affirmed that subacute LBP can be treated successfully with an approach that includes a clinical examination and information to the patient about the nature of the problem to reduce fear and motivate them to resume light activity [10]. Wheeler revealed that chronic LBP is the most expensive benign condition in industrialized countries and the most common cause of activity limitation in persons younger than 45 years [28].

From the World Health Organization's perspective, QoL is a broad concept that incorporates a person's perception of his or her physical health, psychological state, level of independence, social relationships, personal values and beliefs, and interaction with the environment [6]. HRQoL, which has received significant attention in medicine and allied health fields, represents a subset of overall QoL and includes those aspects of QoL that are directly related to an individual's health and perhaps the target of clinical intervention. It has been asserted that one way of capturing the personal and social context of wellness is to use a QoL measure [4]. Health professionals frequently make quality of life judgments when making decisions about the care of patients [15], and the professional view of the expected QoL is often the key factor in determining whether effective treatment for a life-threatening condition will be given or withdrawn [22]. Therefore, it is important to assess the disability status and QoL in persons with LBP to characterize the natural history of the condition, assess treatment effectiveness, and develop an appropriate health and disability policy.

Accordingly, morbidity and prognosis of clinical conditions such as LBP might be differently influenced by its gradients within specific regions of the world. Additionally, the overall impact of LBP which includes physical discomfort, functional limitation and emotional discomfort characteristically affects sufferers' HRQoL $[9,21]$. This is because health has been defined as a state of complete physical, mental and social well-being and not merely the absence of disease or infirmity [29]. Conjointly, individuals with chronic pain have been reported in a study to have lower HRQoL [2]. Also, in another important study, patients with chronic pain had a lower QoL, especially those with LBP and multiple pain localizations [13]. On the other hand, marital status has been reported to be minimally related to $\mathrm{HRQoL}$ in LBP patients in some developed countries [3, 5, 25]. A study also established the significant impact of marital status on HRQoL in LBP patients [1]. QoL is considered to be influenced by several factors including social, cultural and environmental factors. Several demographic and clinical factors are said to influence HRQoL. Some of the implicated clinical factors include physical, psychological, acute or chronic clinical conditions and pain intensity $[9,23,24]$, and the demographic factors are age, gender, ethnicity, marital status and socioeconomic status $[8,12,16]$. It is worth noting that gradients of LBP are never the same across communities in the same country and neither are they the same across countries. For this reason the impact of clinical characteristics of morbidity (CCM) and marital status on HRQoL and contextually on LBP may not be the same as found elsewhere. The study investigated the interface of CCM and marital status with HRQoL among patients with LBP.

\section{Research hypotheses}

The following hypotheses were formulated and tested at the 0.05 alpha level.

1. Marital status will have no significant correlation with HRQoL in patients with LBP.

2. Clinical characteristics of morbidity (CCM) will not significantly associate with HRQoL in patients with LBP.

\section{Material and methods}

\section{Research design}

This was a correlational survey study of the interface of marital status and CCM with HRQoL among LBP patients in Kano metropolis.

\section{Population}

The population for this study included adults with LBP of mechanical origin attending a rehabilitation programme in the three tertiary health institutions in Kano (Aminu Kano Teaching Hospital (AKTH), Murtala Muhammed Specialist Hospital (MMSH), and National Orthopaedic Hospital, Dala (NOH).

\section{Sample size and sampling technique}

A total of 100 subjects with LBP of mechanical origin attending a rehabilitation programme in the abovementioned hospitals participated in this study. They were recruited using the proportionate random sampling technique.

\section{Data collection instruments}

The short form-36 (SF-36), a 36-item tool structured into 8 domains - namely, physical functioning $(\mathrm{PF})$, role limitations due to physical health problems (RP), bodily pain (BP), general health perceptions $(\mathrm{GH})$, vitality (VT), social functioning (SF), role limitation due to emotional problems (RE), and mental health $(\mathrm{MH})$ - was used to assess HRQoL, and also CCM were categorized based on duration of onset of LBP in weeks into acute ( $<6$ weeks), subacute (6-12 weeks) and chronic ( $>12$ weeks). 


\section{Validity and reliability of data collection instrument}

The SF-36 is an internationally validated generic questionnaire, and its reliability was calculated to be 0.84 to $0.91(\mathrm{r}=0.84-0.91)$.

\section{Data collection procedure}

Approval from the Ethical Committees of the abovementioned hospitals was obtained for permission to conduct this study, and the subjects were then recruited consecutively. Informed consent was sought from the subjects, and thereafter the objectives of the study were explained to the respondents. The SF-36 was administered face to face by the researcher, and there was $100 \%$ retrieval. SF36 responses vary from dichotomous (yes or no) to a sixpoint verbal rating scale (ordinal). All items in this tool were scored on a scale of 0 to 100 , with 100 representing the highest level of functioning possible. Aggregate scores were compiled as a percentage of the total points possible. The scores from those questions that address each domain of functional health status were aggregated and then averaged, to arrive at a final score within each of the 8 domains. Also, the duration of onset of LBP of respondents was retrieved through their hospital files to determine their CCM.

\section{Data analysis procedure}

The data obtained from this study were analysed using both descriptive and inferential statistics. The marital status and clinical characteristics of morbidity of participants (acute, subacute and chronic) were summarized using frequencies and percentages, while mean and standard deviation were used to describe domains of HRQoL. The relationship of marital status and CCM with HRQoL was analysed using Pearson's product moment coefficient of correlation. All analyses were performed at the 0.05 alpha level using Statistical Package for the Social Sciences (SPSS, version 20.0).

\section{Results}

The results are presented in Tables 1 and 2.

The results of this study showed that most participants were married $(61 \%)$ and the majority of the participants had chronic LBP (59\%). Also, CCM were found to correlate inversely with all domains of HRQoL except ME, though that was insignificant ( $>00.05$ ), but it may indicate that patients with chronic LBP are prone to have worse HRQoL. However, a proportionate but insignificant relationship was found between marital status and domains of HRQoL except in PF and RP, implying that subjects who are married have better HRQoL.
Table 1. Marital status, $\mathrm{CCM}$ and $\mathrm{HRQ} \mathrm{LL}$ profile of respondents

\begin{tabular}{lcc}
\hline Variables & $\mathrm{n}[\%]$ & $\mathrm{M} \pm \mathrm{SD}$ \\
\hline Marital status & & \\
\hline Married & $61[61]$ & - \\
Single & $39[39]$ & - \\
\hline CCM & & \\
\hline Acute & $23[23]$ & - \\
Subacute & $18[18]$ & - \\
Chronic & $59[59]$ & - \\
\hline HRQoL & & \\
\hline GH & - & $62.59 \pm 23.14$ \\
PF & - & $66.35 \pm 23.49$ \\
RP & - & $53.25 \pm 35.11$ \\
RE & - & $53.91 \pm 38.18$ \\
SF & - & $57.90 \pm 26.90$ \\
BP & - & $51.70 \pm 20.79$ \\
ME & - & $60.79 \pm 20.12$ \\
VT & - & $62.83 \pm 18.52$ \\
\hline
\end{tabular}

$\mathrm{n}$ - frequency within a group or subgroup, $\%$ - percentage within a group or subgroup, $\mathrm{M} \pm \mathrm{SD}-$ mean \pm standard deviation, $\mathrm{CCM}$ - clinical characteristics of morbidity, HRQoL - health-related quality of life, $\mathrm{GH}$ - general health perceptions, $\mathrm{PF}$ - physical functioning, RP - role limitations due to physical health problems, RE - role limitations due to emotional problems, SF - social functioning, BP - bodily pain, $\mathrm{ME}$ - mental health, and VT - vitality

\section{Hypothesis testing}

Hypothesis 1

Marital status will have no significant correlation with HRQoL in patients with LBP.

Findings from this study indicated no significant relationship ( $\boldsymbol{p}>0.05)$ between marital status and all domains of HRQoL. Therefore, the null hypothesis is retained and any relationship noted can be attributed to chance, indicating that marital status is not a predictor of HRQoL in patients with LBP.

\section{Hypothesis 2}

Clinical characteristics of morbidity (CCM) will not associate significantly with HRQoL in patients with LBP.

Clinical characteristics of morbidity were not found to associate significantly $(\boldsymbol{p}>0.05)$ with HRQoL; hence the null hypothesis is retained. This means that chronicity of LBP does not predict HRQoL in subjects with LBP. 
Table 2. Correlations of Respondents' Marital Status (MS) and CCM with Domains of HRQoL

\begin{tabular}{|c|c|c|c|c|c|c|c|c|c|c|}
\hline & MS & $\mathrm{CCM}$ & $\mathrm{GH}$ & $\mathrm{PF}$ & $\mathrm{RP}$ & $\mathrm{RE}$ & $\mathrm{SF}$ & BP & $\mathrm{ME}$ & VT \\
\hline MS & 1 & & & & & & & & & \\
\hline $\mathrm{CCM}$ & $\begin{array}{l}0.149 \\
0.139\end{array}$ & 1 & & & & & & & & \\
\hline $\mathrm{GH}$ & $\begin{array}{l}0.069 \\
0.498\end{array}$ & $\begin{array}{c}-0.078 \\
0.440\end{array}$ & 1 & & & & & & & \\
\hline PF & $\begin{array}{c}-0.110 \\
0.276\end{array}$ & $\begin{array}{c}-0.180 \\
0.073\end{array}$ & $\begin{array}{c}0.646^{*} \\
0.000\end{array}$ & 1 & & & & & & \\
\hline $\mathrm{RP}$ & $\begin{array}{c}-0.028 \\
0.780\end{array}$ & $\begin{array}{c}-0.014 \\
0.886\end{array}$ & $\begin{array}{c}0.452^{*} \\
0.000\end{array}$ & $\begin{array}{c}0.600 * \\
0.000\end{array}$ & 1 & & & & & \\
\hline $\mathrm{RE}$ & $\begin{array}{l}0.037 \\
0.711\end{array}$ & $\begin{array}{c}-0.026 \\
0.796\end{array}$ & $\begin{array}{c}0.500 * \\
0.000\end{array}$ & $\begin{array}{c}0.591^{*} \\
0.000\end{array}$ & $\begin{array}{c}0.780^{*} \\
0.000\end{array}$ & 1 & & & & \\
\hline SF & $\begin{array}{l}0.044 \\
0.660\end{array}$ & $\begin{array}{c}-0.015 \\
0.879\end{array}$ & $\begin{array}{c}0.472 * \\
0.000\end{array}$ & $\begin{array}{c}0.497 * \\
0.000\end{array}$ & $\begin{array}{c}0.684 * \\
0.000\end{array}$ & $\begin{array}{c}0.770^{*} \\
0.000\end{array}$ & 1 & & & \\
\hline BP & $\begin{array}{l}0.036 \\
0.722\end{array}$ & $\begin{array}{c}-0.193 \\
0.055\end{array}$ & $\begin{array}{c}0.370^{*} \\
0.000\end{array}$ & $\begin{array}{c}0.439 * \\
0.000\end{array}$ & $\begin{array}{c}0.528^{*} \\
0.000\end{array}$ & $\begin{array}{c}0.657^{*} \\
0.000\end{array}$ & $\begin{array}{c}0.750^{*} \\
0.000\end{array}$ & 1 & & \\
\hline ME & $\begin{array}{l}0.093 \\
0.356\end{array}$ & $\begin{array}{l}0.027 \\
0.792\end{array}$ & $\begin{array}{c}0.318^{*} \\
0.001\end{array}$ & $\begin{array}{c}0.368^{*} \\
0.000\end{array}$ & $\begin{array}{c}0.492 * \\
0.000\end{array}$ & $\begin{array}{c}0.540^{*} \\
0.000\end{array}$ & $\begin{array}{c}0.591 * \\
0.000\end{array}$ & $\begin{array}{c}0.467^{*} \\
0.000\end{array}$ & 1 & \\
\hline VT & $\begin{array}{l}0.057 \\
0.570 \\
\end{array}$ & $\begin{array}{c}-0.158 \\
0.116 \\
\end{array}$ & $\begin{array}{c}0.431^{*} \\
0.000\end{array}$ & $\begin{array}{c}0.529 * \\
0.000\end{array}$ & $\begin{array}{c}0.540^{*} \\
0.000\end{array}$ & $\begin{array}{c}0.636^{*} \\
0.000\end{array}$ & $\begin{array}{c}0.595^{*} \\
0.000\end{array}$ & $\begin{array}{c}0.539^{*} \\
0.000\end{array}$ & $\begin{array}{c}0.631 * \\
0.000\end{array}$ & 1 \\
\hline
\end{tabular}

MS - marital status, CCM - clinical characteristics of morbidity, GH - general health perceptions, PF - physical functioning, RP - role limitations due to physical health problems, RE - role limitations due to emotional problems, SF - social functioning, BP - bodily pain, ME - mental health, and VT - vitality. Correlation is significant at $0.05 \alpha$ level, critical $r_{0.05,100}=0.1946$.

\section{Discussion}

Marital status was found in this study to have a direct correlation with HRQoL, although it was insignificant, implying that being married would result in a better HRQoL. Being married suggests care and support from spouses. There is continuous support and care for each other; such support could include reminding the spouse of ingestion of medication at the right time, support in settling hospital bills, and reminding about activities to be carried out and those to be avoided; all these would go a long way to improving the HRQoL. Previous studies have reported similar findings, suggesting that marital status influences HRQoL in LBP patients $[8,12,16]$.

The inverse relationship between CCM and HRQoL suggests that having acute LBP is associated with better HRQoL. Chronic LBP is characterized by disabling and devastating lifestyle, and this might suggest why better HRQoL is seen in acute LBP patients in this study. This finding is supported by a study reporting that chronicity is one of the insignificant factors posited to influence delays in seeking treatment and, consequently, to adversely affect HRQoL [27]. Many studies have reported that CCM are significant correlates of HRQoL in patients with LBP $[9,17,21,23,24]$, thereby contrasting with the finding of this study where it was found to be insignificant. These contrasting findings might be a result of variation in study methodology including subject characteristics, differences in measuring instruments of HRQoL, etc.

\section{Conclusions}

Clinical characteristics of morbidity (CCM) and marital status were found to associate disproportionately and proportionately respectively with almost all domains of HRQoL, but they could not determine HRQoL of patients with LBP.

It is expected that the findings of this study:

- May help clinicians during clinical decision making by enabling them to take into consideration the physical, social and mental effects of CCM and marital status in the management of patients with LBP, thereby enhancing clinical outcome.

- Might also serve as an index for the government in healthcare and policy making as it indicates the relationship of CCM and marital status with HRQoL among LBP individuals. 
- May help to establish the most common clinical characteristics of LBP morbidity among people who suffer LBP, thereby enabling preventive measures of the ailment.

\section{Recommendations}

Based on the findings, the following recommendations were made:

1. Clinical characteristics of morbidity (CCM) and marital status must not necessarily be taken into consideration during rehabilitation of low back pain (LBP) patients.

2. Health-related quality of life (HRQoL) measures should be introduced into clinical practice.

3. Clinicians/clinical staff should be trained in using and interpreting the HRQoL measures.

\section{References}

1. Adler L.V., O. Marmot., H. McEwen (2006) A prospective study of the importance of psychological and social factors for the outcome after surgery with slipped lumbar disk operated upon for the first time. Acta Neurochir (Wien), 88: 119-125.

2. Becker N., A.B. Thomsen, A.K. Olsen, P. Sjogren, P. Bech., J. Eriksen (1997) Pain epidemiology and health related quality of life in chronic non-malignant pain patients referred to a Danish multidisciplinary pain center, Pain, 73: 393-400.

3. Bowling A., J. Windsor (2001). Towards the good life: A population survey of dimensions of quality of life. J. Happiness Stud., 2: 5-81.

4. Bowling A. (1995) Measuring disease; a review of quality of life measurement scales. Milton Keyes: Open University Press.

5. Bradford R., D.L. Rutherford, A. John (2002) Quality of life in young people: Rating and factor structure of the quality of life profile-adolescent version. $J$ Adolesc, 25: 261-274.

6. Chandra A., A. Ozturk (2005) In context: Quality of life issues and assessment tools as they relate to patients with chronic nonmalignant pain. Hospital Topics: Research and Perspectives on Healthcare, 83: 33-37.

7. Global Burden of Disease (2010) One year lived with disability (YLDs) for 1160 sequelae of 289 diseases and injuries 1990-2010: a systematic analysis for the Global Burden of Disease Study. Lancet, 15: 380 (9859), 216396. DOI: 10.1016/S0140-6736(12)61729-2.

8. Hijmans C.T., K. Fijnvandraat, J. Oosterlaan, H. Heijboer, M. Peters, M.A. Grootenhius, (2010) Double disadvantage: A case study on health related quality of life in children with sickle cell disease. Health Quality of Life Outcome, 8: 121.
9. Horng Y.S., Y.H. Hwang, H.C. Wu, H.W. Liang, Y. Jang, F.C. Twu (2005) Predicting health-related quality of life in patients with low back pain. Spine, 30(5): 551-555.

10. Indahl A., L. Velund, O. Reikeraas (1995) Good prognosis for low back pain when left untampered: a randomized clinical trial. Spine, 20: 473-477.

11. Klenerman L., P.D. Slade, I.M. Stanley (1995) The prediction of chronicity in patients with an acute attach of low back pain in general practice setting. Spine, 20: 478-484.

12. Kwon M.A., W.S. Shin, M.H. Kin, M.S. Gwak, T.S. Hahm, G.S. Kim (2006) A correlation between low back pain and associated factors: A study involving 772 patients who had undergone general physical examination. J. Korean Med. Sci., 21: 1086-1091.

13. Lame I.E., M.L.Peters, J.W.S. Vlaeyen, M.V. Kleef, J. Patijn (2005) Quality of life in chronic pain is more associated with beliefs about pain, than with pain intensity. Eur. J. Pain, 9: 15-24.

14. Loney P., P. Stratford (1999). The prevalence of low back pain in adult: A methodological review of the literature. Physical Therapy, 79(4): 384-396.

15. Manara A.R., J.A. Pittman, F.E. Braddon (1998) Reasons for withdrawing treatment in patient receiving intensive care. Anaesthesia, 53: 523-528.

16. Mansour M.E., U. Kotagal, B. Rose, H.M. Brewer, RoyChaudhury (2003) Health-related quality of life in urban elementary school children. Pediatrics, 111: 1372-1381.

17. Mantyselka P.T., J.H. Turunen, R.S. Ahonen, E.A. Kumpusalo (2003) Chronic pain and poor self-rated health. JAMA, 290(18) : 2435-2442.

18. Naidoo V. (2009) Physiotherapy modalities used in the management of chronic lowback pain. University of the Witwatersrand, Johannesburg.

19. Niemisto L., T. Lahtinen-Suopanki, P. Rissanen, K.A. Lindgren, S. Sarna, H. Hurri (2003) A randomized trial of combined manipulation, stabilization exercises, and physician consultation compared to physician consultation alone for chronic low back pain. Spine, 28(19): 2185-2191.

20. Owoeye I. M. Eze (2005) Executive health: maintenance and management. Lagos: University of Lagos press.

21. Patrick D.L., R.A. Deyo, S.J. Atlas (1995) Assessing health-related quality of life in patients with sciatica. Spine, 20(1): 899-908.

22. Pellegrino E.D. (2000) Decisions to withdraw life-sustaining treatment: A moral algorithm. JAMA, 290(18): 283-1067.

23. Pellise F., F. Balague, L. Rajmil, C. Cedraschi, M. Aguirre, C.G. Fontecha (2009) Prevalence of low back pain and its effect on health-related quality of life in adolescents. Arch. Pediatr. Adolesc. Med., 163(1): 65-71.

24. Sherman E.M.S., Y.S. Griffiths, S. Akdag, M.B. Connolly, D.J. Slick, S. Wiebe (2007) Sociodemographic correlates of health related quality of life in pediatric epilepsy. Epilepsy and Behavior, 12: 96-101. 
25. Trompenaar F.J., E.D. Masthoff, G.L.Van-Heek, P.P. Hodiamont, J. De Vrie (2005) Relationships between demographic variables and quality of life in a population of Dutch adult psychiatric patients. Soc. Psychiatry Epidemiol., 40: 588-594.

26. Tulder M.V., A. Becker, A. Bekkering, A. Breen, G.T. Gil (2004) Guidelines for the management of acute low back pain in primary care, European Commission.

27. Van-Servellen G., B. Chang, E. Lombardi (2002) Acculturation, socioeconomic vulnerability, and quality of life in Spanish-speaking and bilingual Latino HIV-infected men and women. West. J. Nurs. Res., 24: 246-263.
28. Wheeler A.H. (2002) Chronic lumbar spine and radicular pain, pathology and treatment. Spine, 6(2): 79-105.

29. World Health Organization (2001) International Classification of Functioning, Disability and Health. Geneva, Switzerland: WHO.

\section{Received 11.10.2016 \\ Accepted 02.12.2016}

(C) University of Physical Education, Warsaw, Poland 